\title{
Acrocardiofacial syndrome
}

INSERM

\section{Source}

INSERM. (1999). Orphanet: an online rare disease and orphan drug data base.

Acrocardiofacial syndrome. ORPHA:2008

Acro-cardio-facial syndrome (ACFS) is a rare genetic disorder characterized by split-

hand/split-foot malformation (SHFM), facial anomalies, cleft lip/palate, congenital heart defect (CHD), genital anomalies, and intellectual deficit. 